\title{
LA VALORACIÓN DEL DOCENTE UNIVERSITARIO EN ENTORNOS VIRTUALES: ALGUNOS DESCRIPTORES CLAVES
}

\author{
Irama F. García V. ${ }^{1}$ \\ Universidad Central de Venezuela - Venezuela \\ irama.garcia@gmail.com \\ Rosa Amaro ${ }^{2}$ \\ Universidad Central de Venezuela - Venezuela \\ rosant34@gmail.com \\ Carmen Brioli ${ }^{3}$ \\ Universidad Central de Venezuela - Venezuela \\ brioli.carmenalicia@gmail.com
}

\begin{abstract}
Recepción: 04/08/2012
Evaluación: 29/09/2012

Aceptación: 15/11/2012

Artículo de investigación científica y tecnológica

doi: http://dx.doi.org/10.9757/Rhela.19.09
\end{abstract}

\section{RESUMEN}

El desarrollo de la educación a distancia a través de la virtualidad, cada vez adquiere mayor importancia en la UCV lo cual se refleja en el interés de algunos docentes por migrar sus cursos de pregrado de la presencialidad a la virtualidad, muchas veces desconociendo la complejidad que comporta desde una perspectiva pedagógica, el desarrollo de experiencias formativas en entornos virtuales de enseñanza y aprendizaje. En esta investigación se planteó valorar las competencias del docente de la UCV, para diseñar y desarrollar experiencias formativas en entornos

\footnotetext{
1 Docente de la Facultad de Humanidades y Odontología de la Universidad Central de Venezuela, Doctora en Educación, mención tecnología instruccional y educación a distancia (NOVA, Southeastern University, USA). Magíster Scientiarium en Educación, mención orientación de la Universidad Central de Venezuela. Licenciada en Educación, mención orientación de la Universidad Central de Venezuela.

2 Docente de tiempo completo de la Universidad Central de Venezuela. Licenciada en Educación, especialista en Formación Virtual y Educación a Distancia. Magíster en Educación Superior de la USB, Magíster en Tecnología Educativa de la Universidad de Salamanca-OEI, España) y Doctora en Ciencias de la Educación.

3 Venezolana. Licenciada en Idiomas Modernos, Mención Docencia (Universidad Metropolitana, Caracas). Estudios de Especialización en Tecnología Educativa (Universidad Central de Venezuela). Magíster en Educación, Mención Tecnologías de la Información y la Comunicación (2006, Universidad Central de Venezuela). Candidata a Doctora en Educación de la Universidad Nacional de Educación a Distancia (Madrid- España) en el Programa Modelos Didácticos, Interculturalidad y aplicación de las Tecnologías de la Información y la Comunicación en las Instituciones Educativas). Miembro del personal docente y de investigación (Escalafón Asociado) del Departamento de Lengua y Comunicación de la Escuela de Educación, Facultad de Humanidades y Educación, Universidad Central de Venezuela.
} 
virtuales de enseñanza y aprendizaje en el nivel de pregrado de la UCV, de calidad y con cierta garantía de éxito.

Esta valoración se hizo a partir de los resultados del análisis de las experiencias que se llevan a cabo actualmente en algunas facultades y escuelas de la UCV, en contraste con el referente teórico elaborado como producto de la revisión teórica sobre el tema, el perfil de competencia y los descriptores considerados claves. Los resultados permitirán en el corto plazo, la formulación de planes de acción orientados a la superación de

\section{UNIVERSITY PROFESSOR'S COMPETENCES PROFILE IN ON-LINE ENVIRONMENTS: SOME KEY DESCRIPTORS}

\begin{abstract}
The development on Distance Learning through on-line tools is becoming increasingly important at the UCV. This is shown by the interest of some professors on changing their undergraduate programs form classroom education to distance learning, most or the times not knowing the complexity that implies form the pedagogical viewpoint the development of educational experiences in on-line environments of teaching and learning.
\end{abstract}

The aim of this study was to assess the competences of the professor at the UCV in order to develop high quality and certain success guarantee-based educational experiences in on-line environments of teaching and learning concerning undergraduate level at the University.

This assessment was carried out based on the results of the analysis regarding the current experiences in some of the schools of the UCV, compared to the theoretical model that resulted from the theoretical review of the topic, competence profile and descriptors considered as imperatives.

The results will allow in the short term, the draw up of action plans faced to overcome detected weaknesses and protect the strengths, with a view to increase educational experiences from the on-line model at the UCV, without leaving aside the criterion of excellence. Furthermore, las debilidades detectadas y la potenciación de las fortalezas, con miras a incrementar las experiencias formativas desde el paradigma de la virtualidad en la UCV, con criterio de excelencia. El estudio además podría aportar insumos para la elaboración posterior de un plan de formación docente en el área, en correspondencia con las políticas del Sistema de Educación a Distancia de la UCV. (SEDUCV).

Palabras clave: Revista Historia de la Educación Latinoamericana, enseñanza a distancia, capacitación, desarrollo de competencias.

\section{A VALORIZAÇÃO DO DOCENTE UNIVERSITÁRIO NOS AMBIENTES VIRTUAIS: ALGUNS CONCEITOS FUNDAMENTAIS}

\section{RESUMO}

O desenvolvimento da Educação à Distância por meio da virtualidade adquire cada vez maior importância na UCV, o que se reflete no interesse de alguns docentes por migrar seus cursos de graduação presenciais para a virtualidade, muitas vezes desconhecendo a complexidade existente a partir de uma perspectiva pedagógica, o desenvolvimento de experiências formativas nos ambientes virtuais de ensino e aprendizagem. Esta pesquisa procurou valorar as competências do docente da UCV, para projetar e desenvolver experiências formativas nos ambientes virtuais ensino e aprendizagem, no nível da graduação da UCV, com qualidade e com garantia de êxito.

Esta valoração foi feita a partir dos resultados da análise das experiências que se levam a cabo atualmente em algumas faculdade e escolas da UCV, em contraste com o referencial teórico elaborado como produto da revisão teórica sobre o tema, o perfil de competência e os conceitos considerados centrais.

Os resultados permitirão, num curto prazo, a formulação de planos de ação orientados à superação das debilidades detectadas e a potencialização das fortalezas, com vistas a incrementar as experiências formativas a partir do paradigma da virtualidade na UCV, com critério de excelência. Ademais, o estudo poderia contribuir com insumos para a elaboração posterior de um plano de formação docente na área, em correspondência 
La valoración del docente universitario en entornos virtuales: algunos descriptores claves

the study could provide elements to a future development of a teacher training plan in the area, pursuant to the policies of the Distance Learning System of the UCV (SEDUCV in Spanish).

Key words: History of Latin American Education Journal, distance learning, training, development of competences. com as políticas do Sistema de Educação à Distância da UCV. (SEDUCV).

Palavras-chave: Revista História da Educação Latino-americana, Ensino à Distância, capacitação, desenvolvimento de competências.

\section{INTRODUCCIÓN}

La educación superior se concibe como un proceso dinámico y cambiante que se debe a las necesidades del desarrollo del país, el avance científico y tecnológico y a las necesidades del estudiante adulto para continuar ampliando su formación humana y científica de alto nivel, de modo que la internalice en forma crítica y la proyecte en forma activa en función de la transformación y el perfeccionamiento de la realidad en la cual se desenvuelve.

Es por ello que la formación permanente y los recursos que se destinan resultan claves para asegurar los procesos de cambio y de innovación requeridos para la mejora continuada de los entornos virtuales de enseñanza y aprendizaje, que actualmente resultan complejos y sin regulación.

La tecnología con el fundamento pedagógico adecuado y un diseño pertinente, puede potenciar los programas formativos en la educación superior y en otros ámbitos académicos que se desarrollan a través de esta modalidad. Así lo resalta Luján (s/f) quien plantea que lo que hace la diferencia en cuanto a la calidad en la enseñanza en línea sigue siendo la pedagogía, la didáctica y, fundamentalmente, la participación de un docente de probada experiencia e idoneidad.

\footnotetext{
Sin embargo, en otros espacios hemos señalado que ... el desempeño docente en entornos de enseñanza y de aprendizaje de calidad, a distancia y a través de la telemática, plantea las mismas exigencias didácticas y pedagógicas que la modalidad presencial, en cuanto a concebir, diseñar, ejecutar, controlar y evaluar el proceso instruccional que se lleva a cabo. Por tanto, la calidad que se logre, dependerá de un ejercicio adecuado y responsable del docente, tanto o más que en la modalidad presencial ${ }^{4}$.
}

4 Rosa Amaro de Chacin, La docencia telemática ¿una nueva docencia? (Orlando: 2009), 65. 
Es por ello que estos entornos deben ser gestionados por un docente competente para desempeñarse adecuadamente en espacios sociales mediados por las tecnologías, que hoy parece ser una gran expectativa para el cambio en la enseñanza universitaria. Este cambio se proyecta en un desarrollo personal y profesional en el cual el docente debe implicarse para introducir innovaciones.

Sin lugar a dudas, el docente debe pasar de transmisor de información a guía del proceso de aprendizaje, convertirse en un motivador y facilitador de recursos, diseñador de nuevos entornos de aprendizaje con TIC, adaptador y productor de materiales en distintos formatos, y evaluador de los procesos que se desarrollan en estos nuevos entornos, pero ello no implica una "nueva concepción del docente". Se trata más bien de reconocer que esta perspectiva de la enseñanza ofrece nuevas oportunidades para lograr un verdadero y significativo aprendizaje con otros medios de facilitación ${ }^{5}$.

El desarrollo de estas competencias, no solo debe abordarse desde el punto de vista tecnológico, sino que fundamentalmente debe ser de carácter pedagógico.

La formación es una herramienta estratégica para el logro de las metas establecidas, que debe proveer cierta garantía de calidad y eficiencia en los procesos y resultados. Por ello, la insistencia en desarrollar las competencias mínimas para gestionar entornos de aprendizaje por cuanto:

\section{... la telemática plantea opciones adecuadas y pertinentes a la demanda educativa de hoy pero también requiere un desempeño docente profesional y responsable como garantía de calidad del proceso didáctico que se lleva a cabo en esta modalidad, y esto conlleva a espacios formativos con una concepción de la enseñanza apropiada, que posibilite la incorporación de las TIC a la práctica didáctica-curricular, sustentada y referenciada convenientemente en la didáctica y la pedagogía ${ }^{6}$.}

\section{Las competencias docentes en los Entornos Virtuales de Enseñanza y Aprendizaje}

Para participar en este espacio social concebido como un espacio para la interacción, cuya importancia se ha incrementado en los últimos tiem-

Rosa Amaro de Chacin, La docencia telemática ¿una nueva docencia? (Orlando: 2009), 267.

De Chacin, La docencia telemática, 272. 
La valoración del docente universitario en entornos virtuales: algunos descriptores claves

pos, se requieren ciertas competencias, tanto por parte de los tutores - moderadores como por los teleaprendientes que garanticen la calidad de estos entornos virtuales. En tal sentido, se comparte lo señalado por Imbernon y otros en cuanto a que:

Debemos tener en cuenta que, para el desarrollo de competencias en el alumnado, los docentes universitarios también deben poseer y saber desarrollar una serie de competencias (llamémoslas competencias pedagógico - didácticas) que permitan facilitar los procesos de aprendizaje autónomos y significativos; los profesores, por tanto, también deben saber, conocer, seleccionar, utilizar, evaluar, perfeccionar y recrear o crear estrategias de intervención didácticas efectivas en un contexto definido por las TIC...?

Es por ello que hoy se plantea como una exigencia, la formación de los docentes universitarios que se desenvuelven o pretenden desempeñarse "competentemente" en estos escenarios, y esto implica no solo aplicar convenientemente la tecnología a la educación, sino diseñar y construir con propiedad el espacio telemático, al igual que; elaborar instrumentos educativos electrónicos. Sin embargo, son pocos o nulos los escenarios específicamente diseñados para la educación en este nuevo espacio social.

Por esta razón se plantea como una necesidad inmediata, no solo crear los escenarios educativos telemáticos sino formar a los agentes responsables de diseñar acciones educativas en las que se considere la flexibilidad del tiempo y otros materiales educativos, y llevarlas a cabo. De este modo, podríamos aproximarnos a una "didáctica del entorno virtual". En lugar del cuaderno y los libros de texto, los materiales del aula virtual están formados por la pantalla, el ratón, el teclado, el material multimedia, la pizarra electrónica y el software entre otros ${ }^{8}$. Esto implica cierta preparación que propicie cambios que deben ser regulados y monitoreados para garantizar su calidad y excelencia.

\section{Construcción del perfil de competencias del docente universita- rio para la enseñanza en EVEA}

La mayoría de las clasificaciones presentadas, focaliza como aspecto sustancial, lo concerniente al componente pedagógico y didáctico, en vir-

F. Imbernon et al., Análisis y propuestas de competencias docentes universitarias para el desarrollo del aprendizaje significativo del alumnado a través del e-learning y el b-learning. (2008), 28. http://tecnologiaedu.us.es/nweb/ htm/pdf/EA20070049_Dr_Francisco_Imbernon.pdf.

8 R. E. Bello, Educación virtual: aulas sin paredes. (Monografía), 2005. http://www.educar.org/articulos/educacionvirtual.asp. 
tud de que las principales funciones que tiene el profesorado en un entorno virtual es el diseño y el desarrollo de ambientes de aprendizaje. La competencia en la enseñanza, desde la perspectiva que se asume en este trabajo, contempla básicamente -sin pretender excluir la importancia de otros aspectos- lo relacionado con el diseño y la e-moderación, aspectos en los cuales nos centraremos por considerar que son claves en la dimensión didáctica de los procesos de enseñanza y aprendizaje en entornos virtuales. El docente que desarrolla su acción en un EVEA tal como aquí se concibe, interviene en todas las fases del proceso formativo por lo que debería ser el más calificado para diseñar, gestionar y evaluar esta clase de formación lo cual implica adquirir el conjunto de competencias que aseguren un proceso de calidad.

Es por ello que se considera imperativo que los docentes se formen para minimizar los riesgos que conlleva migrar la práctica tradicional y reproductora, a los entornos de formación virtual. De modo que, en correspondencia con la intencionalidad pretendida en este apartado y con base en el referente teórico abordado en páginas anteriores ${ }^{9}$, se presenta un perfil por competencias especifico (según la clasificación de TUNING) centrado en el ámbito de la didáctica, atendiendo a las particularidades de la docencia universitaria y la enseñanza en entornos virtuales que pueda servir de referencia a programas de formación y actualización del docente universitario. La construcción de un perfil específico que además del componente disciplinar apunte al componente tecnológico y didáctico se justifica a la luz del modelo teórico presentado por Mishra y Koehler ${ }^{10}$ al puntualizar en los conocimientos del contenido o disciplina, de la pedagogía y de la tecnología a partir de la integración de lo disciplinar lo tecnológico y lo didáctico.

En esta clasificación se considera lo señalado por Escudero ${ }^{11}$ en cuanto a que además de contemplar los dominios referidos a lo tecnológico y lo didáctico, se toma en cuenta un tercer dominio referido a competencias relacionadas con la reflexión y el análisis crítico de la experiencia desarrollada individualmente o en

\footnotetext{
9 Oliveira, Cervera y Martí, Learning as representation and representation as learning: a theoretical framework for teacher knowledge in the digital age. (Chesapeake: AACE, 2009).

10 Mishra y Koehler, Conocimiento disciplinar, pedagógico y tecnológico (Technology Pedagogical Content Knowledge TPACK) (2006). http://www.wiziq.com/tutorial/71616-Mishra-amp-Koehler.

11 J. M. Escudero, La formación del profesorado y la garantía del derecho a una buena educación para todos. (Barcelona: Octaedro, 2006).
} 
La valoración del docente universitario en entornos virtuales: algunos descriptores claves

forma colectiva, lo cual apunta a la autovaloración y retroalimentación de las experiencias formativas que el docente desarrolla especialmente (más no exclusivamente) en los entornos virtuales.

Por otra parte, Marcelo ${ }^{12}$ aporta valiosos elementos para la caracterización de los dominios competenciales, particularmente útiles en la operacionalización del perfil que se presentan especialmente en lo referido a lo tecnológico y al diseño (incluye además tutorial y gestión) y se enriquece con las competencias que Gomes de Oliveira ${ }^{13}$ ubica en el dominio personal.

Por lo antes señalado, en el perfil que se presenta, se puntualiza en el componente didáctico y dentro de este se aborda lo relacionado con las competencias en diseño y en la e-moderación.

Según la UNESCO (2008), citada por Bustamante ${ }^{14}$, existe un conjunto de estándares de conocimientos y competencias en TIC que los docentes del siglo XXI deben conocer y dominar para aplicarlas con un fin determinado.

En tal sentido la autora precitada agrupa estos conocimientos y competencias sobre TICS en tres (3) categorías las cuales mencionamos a continuación:

- Conocimientos sobre una variedad de aplicaciones y herramientas tecnológicas.

Estos conocimientos deben permitir al docente el saber y poder usar, con suficiente flexibilidad, las diversas aplicaciones y herramientas tecnológicas en una variedad de situaciones de enseñanza-aprendizaje basadas principalmente en la metodología de trabajo por proyectos y estrategias para la solución de problemas. Se destaca que estos conocimientos deben abarcar principalmente la selección de recursos tecnológicos que faciliten el acceso a la información y promuevan la comunicación y la colaboración con expertos exter-

12 Marcelo G., Prácticas de E-learning. Las nuevas competencias en e-learning: ¿Qué formación necesitan los profesionales de e-learning?(España: Octaedro, 2006).

13 De Oliveira Gomes, La acción docente en la educación a distancia. Competencias para la mediación en red. (2007). http://www.usal.es/-teoriaeducacion/rev_numero_08_02/n8_02_oliveira.pdf

$14 \mathrm{H}$. Bustamante, Redes digitales e indicadores de calidad educativa para el caso de un programa de actualización docente (México: Universidad Nacional Autónoma de México, (s/f)), http://www.distancia.unam.mx/foroeducativo.html. 
nos con el fin de analizar conjuntamente los problemas planteados en los proyectos y sugerir propuestas para su solución. Para el logro de lo antes planteado sugiere que los docentes deben ser capaces de crear proyectos de aula a ser llevados a cabo de forma individual o en grupo, además de hacer un seguimiento constante de los mismos en su etapa de realización.

- Competencias para crear ambientes de aprendizaje basados en el uso de las TIC.

Estos ambientes deben estar centrados en el estudiante y el uso de las TIC debe ser flexible y promover la cooperación.

- Competencias para propiciar su autoformación profesional a través de las TIC.

El uso de las TIC como respaldo a la propia formación profesional del docente, requiere a la vez que este posea competencias relacionadas con: el saber crear o elaborar proyectos complejos; saber cómo utilizar las redes para acceder a información, saber utilizar las redes para comunicarse con otros colegas y expertos y estar en capacidad de colaborar con sus pares.

Tal y como se puede observar en las competencias antes mencionadas, se hace difícil desvincular los aspectos tecnológicos de los pedagógicos. Esto se puede evidenciar claramente cuando la autora, basándose en lo establecido por la UNESCO ${ }^{15}$, menciona las tres categorías de competencias que denomina tecnológicas, conjuntamente con aspectos asociados a la enseñanza y el aprendizaje en el marco de un enfoque cognitivo constructivista. En tal sentido, se vinculan las competencias tecnológicas con aspectos didácticos tales como:

- La metodología de trabajo por proyectos.

- Las estrategias didácticas para la solución de problemas.

- El enfoque de aprendizaje cooperativo.

- El enfoque o teoría de enseñanza centrada en el estudiante.

- La creación de ambientes flexibles de enseñanza.

- La autoformación del mismo docente no solo a través de un mayor acceso a la información sino también mediante el trabajo colaborativo con sus colegas.

Se puede concluir entonces, que la autora precitada enfatiza la relación que debe existir entre la dimensión informática y telemática (TIC) y su

15 UNESCO. Estándares de competencias en TIC para docentes. (2008). http://www.iesalc.unesco.org.ve 
La valoración del docente universitario en entornos virtuales:

algunos descriptores claves

uso en el autoaprendizaje y la docencia, en otras palabras, el docente debe poseer un conocimiento tanto instrumental como didáctico de las diferentes aplicaciones tecnológicas con el fin de ser capaz de utilizarlas tanto en su formación profesional como en el proceso de enseñanza y aprendizaje con su grupo de alumnos.

\section{Competencias en diseño instruccional para los EVEA}

En términos generales, una competencia en diseño instruccional se define como la habilidad para tomar decisiones adecuadas y sustentadas desde el punto de vista didáctico y pedagógico y traducirlas en la creación de programas de formación de excelencia y calidad en cualquier ámbito de intervención. Tal como se ha señalado en páginas anteriores, una competencia es dinámica, fluida y compleja que se manifiesta valga la redundancia, en un acto real "competente" es decir con el más alto nivel de eficacia y eficiencia, por ello conjuntamente con Marcelo, se asume que el diseñador debe:

... mostrar capacidad para seleccionar la metodología más adecuada para llevar a cabo el curso y realizar tareas de seguimiento y supervisión del mismo manteniendo de estforma una información actualizada y un conocimiento del éxito de la acción formativa ${ }^{16}$.

¿Qué hace a un docente-teleformador, competente en diseño?

Sin duda, el docente que desarrolla acciones formativas en línea debe tener el conocimiento que lo hace potencialmente capaz para tomar las decisiones más acertadas que se han de traducir en la práctica del diseño instruccional y la disposición para realizarlo. Por ello, tal como aquí se concibe, la competencia integra las dimensiones cognitiva-reflexiva, activo-creativas y afectivas-comunicativas.

Desde esta perspectiva y aun corriendo el riesgo de no ser todo lo exhaustivo posible pues no es la intencionalidad en este espacio, se señalan a continuación algunas dimensiones que integran la competencia para elaborar diseños de calidad en programas de formación virtual.

A partir del enfoque en que se sustenta este trabajo, el teleformador competente en diseño:

$\overline{16}$ G. Salmon, Prácticas de E-learning, 46. 
- Reconoce las bases teóricas de la Educación a Distancia desde el paradigma de la virtualidad, y los principios de la enseñanza y el aprendizaje adulto.

- Conceptualiza el diseño en entornos virtuales como proceso dinámico, flexible y abierto que admite modificaciones en función de las necesidades del alumnado y que conjuga los aspectos conceptuales y los aspectos constitutivos de los EVEA considerado como un proceso.

- Realiza un diagnóstico de necesidades de formación, empleando procedimientos adecuados. En este sentido debe conocer algunas estrategias de análisis que permitan detectar y recoger las necesidades existentes para poder así dar una adecuada respuesta a las mismas.

- Caracteriza la acción formativa atendiendo a su referente contextual y teórico que permite, por una parte, la clara justificación desde el punto de vista del área de conocimiento, la modalidad y la necesidad instruccional u organizacional a la cual responde la acción formativa. Por otra parte, fundamente teóricamente la propuesta formativa virtual.

- Explora la situación inicial de los participantes, empleando procedimientos adecuados para diagnosticarlas.

- Formula objetivos de la acción formativa, de manera clara, identificando los tipos de objetivos que se quieren lograr atendiendo al tipo de aprendizaje que se promueve.

- Selecciona los contenidos en correspondencia con los objetivos y los principios de usabilidad que la Web requiere y los organiza en una secuencia apropiada, empleando una estructura adecuada (ramificada, jerárquica en red y en torno a problemas y casos.) que permita la navegación e interacción con páginas Web externas, artículos electrónicos, libros electrónicos, etc.

- Conoce y selecciona variadas herramientas tecnológicas en función de los objetivos previstos, que favorecen el aprendizaje significativo, interactivo y colaborativo.

- Selecciona y organiza estrategias de facilitación en correspondencia con los objetivos programados y la orientación teórica asumida, considerando los espacios de socialización, comunicación e interacción. 
La valoración del docente universitario en entornos virtuales:

algunos descriptores claves

- Selecciona y organiza estrategias de aprendizaje en correspondencia con los objetivos programados y la orientación teórica asumida considerando las potencialidades de las herramientas tecnológicas disponibles.

- Selecciona y organiza estrategias evaluativas en correspondencia con los objetivos programados y la orientación teórica asumida.

- Contempla actividades de seguimiento, supervisión y evaluación de la propuesta formativa con el objeto de mantener la información actualizada, asegurar el buen funcionamiento del proceso y retroalimentarlo para incrementar su calidad y eficiencia.

- Determina el tiempo.

- Trabaja en equipo con otros especialistas.

La gestión planificada y organizada de la enseñanza en la modalidad a distancia y virtual, -tal como aquí se concibe- se fundamente en la pedagogía problematizadora lo cual plantea la necesidad de:

Reflexionar sobre las operaciones que intervienen en la programación de la enseñanza, es decir, tener una práctica consciente, intencionada de decisiones, iniciativas, actividades con una justificación explícita, conocida y asumida por los ejecutantes de la enseñanza. La práctica de la enseñanza necesita un cierto orden dentro de lo que puede y debe tener cabida el criterio de flexibilidad - indeterminación. Ese orden supone una visión previa de lo que se va a realizar.

Intervenir en la realidad con la finalidad de propiciar cambios significativos en los participantes tanto en los aspectos cognitivos como sociales y esto supone un adecuado desempeño pedagógico y social (entre otros por parte del docente tutor o moderador en espacios virtuales, clave en la gestión y dinamización del grupo y en la motivación y creación de un ambiente amistoso y socialmente positivo que permita generar una comunidad de aprendizaje con la intención de promover la interacción, el auto-aprendizaje y la construcción del conocimiento compartido.

Aun cuando en la literatura, algunos autores ${ }^{17}$ definen la e-moderación como un rol del tutor, en este trabajo, se entienden los conceptos de "tutor

7 J. Silva, El rol moderador del tutor en la conferencia mediada por computador (Islas Baleares: EDUTEC, 2004), http://www.uib.es/depart/gte/edutec-e/revelec17/silva_16a.htm 
en línea" y "e-moderador" como sinónimos aunque este último -popularizado por Salmon ${ }^{18}$ - es el que se emplea en el contexto de esta investigación y se le atribuye la importancia de su intervención (guía y ayuda pedagógica) al proceso de la socioconstrucción del conocimiento compartido para lo cual debe crear en el aspecto social, un ambiente amistoso y socialmente positivo que sea propicio para el desarrollo de un ambiente de aprendizaje positivo en comunidad. En el aspecto intelectual debe enfocar los puntos fundamentales, recapitular y evaluar las intervenciones. Por ello, otra de las competencias básicas del docente en los EVEA es la e-moderación, donde se materializa la comunicación didáctica.

\section{Competencias para la e-moderación}

Un diseño adecuado de un EVEA está asociado al éxito del desarrollo de la experiencia formativa en línea; sin embargo, la acción del docente a lo largo de la implementación de este diseño puede ser un factor que dificulte o promueva el aprendizaje, por ello la conveniencia de atender a las competencias que además del diseño, garanticen una buena moderación virtual.

En términos generales, una competencia en e-moderación se define como la habilidad para dinamizar, orientar y dirigir en línea un grupo de personas a través de herramientas comunicacionales síncronas y asíncronas.

Salmon ${ }^{19}$ propone cinco etapas en el proceso de la e-moderación, que deben considerarse en la implantación de un curso en línea. Estas etapas se traducen en la estrategia de facilitación contemplada en el diseño y sugieren la conveniencia de desarrollar ciertas actividades que contribuyen a prevenir las causas que originan la frustración del estudiante en línea de acuerdo con los resultados de la investigación desarrollada por Borges ${ }^{20}$, de modo que las actividades formuladas en el diseño deben ser coherentes con las etapas de la e-moderación planteadas, en el caso específico que nos ocupa, las etapas propuestas por Salmon ${ }^{21}$.

\footnotetext{
G. Salmon, E-actividades. El factor clave para una formación en linea activa. (Barcelona: UOC, 2002). G. Salmon, E-actividades.

20 Borges F. La frustración del estudiante en línea. Causas y acciones preventivas. (2005). http://www.uoc.edu/digithum/7/dt/esp/borges.pdf

21 Salmon G. E-actividades. El factor clave para una formación en línea activa. (Barcelona: UOC, 2002).
} 
La valoración del docente universitario en entornos virtuales: algunos descriptores claves

La autora precitada, propone un modelo de cinco 5 etapas tal como se describe a continuación:

Etapa 1: Acceso y motivación: Durante esta primera etapa es esencial acceder al ambiente y poder adquirir las habilidades para utilizar el computador, como requisito previo para la participación en la experiencia de aprendizaje virtual.

Etapa 2: Socialización: La segunda etapa tiene que ver con el hecho de que los participantes se identifican en línea y comienzan a interactuar. Esto requiere de una intervención apropiada por parte del E-moderador para favorecer la comunicación entre pares estableciendo una comunidad de aprendizaje en la que trabajan en forma conjunta.

Etapa 3: Compartir información: En esta etapa comienza a producirse el intercambio de información y esta se caracteriza por el gran volumen de aportes que genera la interacción. En este proceso el e-moderador ofrece ayuda y direccionamiento a los participantes.

Etapa 4: Construcción de conocimiento: Es la etapa en la que el grupo se relaciona a través de la discusión, y participa en la construcción del conocimiento.

Etapa 5: Desarrollo: Los participantes se vuelven más críticos con el ambiente tecnológico y su funcionamiento, así como el actuar del tutor, lo que puede dificultar en algunos casos la negociación. Es una etapa de construcción individual del conocimiento.

En este apartado se plantean los indicadores que permiten apreciar cada una de las etapas de la e-moderación según Salmon ${ }^{22}$ los cuales hemos considerado como dimensiones de la competencia, complementadas con las competencias que señala $\mathrm{Marcelo}^{23}$, como se detalla a continuación:

\section{$1^{\circ}$ Acceso y motivación}

- Proporciona mensaje inicial de bienvenida.

- Conoce y se familiariza con los alumnos, sus características y condiciones desde el comienzo del curso.

\footnotetext{
Salmon G. E-actividades. El factor clave para una formación en línea activa. (Barcelona: UOC, 2002) 186.

23 Marcelo G. Prácticas de e-learning. Las nuevas competencias en e-learning: ¿Qué formación necesitan los profesionales de e-learning? (Espańa: Octaedro, 2006).
} 
- Ayuda a los alumnos a familiarizarse con la plataforma, con los contenidos y con las herramientas de comunicación.

- Explica lo que se espera de los participantes.

\section{$2^{\circ}$ Socialización}

- Promueve la socialización en línea para que los participantes intervengan con frecuencia.

- Proporciona retroalimentación oportunamente.

- Evidencia una adecuada capacidad de respuesta ante los problemas planteados.

- Reconoce las contribuciones de los participantes.

- Anima a la intervención en línea.

- Envía mensajes de apoyo que animan y evitan la sensación de soledad y aislamiento.

\section{$3^{\circ}$ Intercambio de información}

- Interactúa con los participantes empleando los medios a su alcance (chat, correo, teléfono, videoconferencia, etc.).

- Interactúa con el contenido.

- Proporciona consejos, sugerencias y aclara dudas sobre el contenido y metodología.

- Promueve la lectura del material.

- Convoca y modera sesiones de chat para aclarar dudas.

- Promueve las respuestas de los alumnos a las dudas planteadas por los compañeros.

- Incluye resúmenes o plenarias.

\section{$4^{\circ}$ Construcción de conocimiento}

- Promueve el diálogo y la colaboración.

- Contempla actividades relacionadas con el tópico de la semana.

- Proporciona retroalimentación en forma individual y grupal destacando los aspectos positivos y negativos de sus trabajos y planteando alternativas de mejora. 
La valoración del docente universitario en entornos virtuales:

algunos descriptores claves

- Proporciona un feedback positivo, así como un seguimiento de la evolución de su aprendizaje.

- Clausura el debate.

\section{$5^{\circ}$ Desarrollo}

- Pide a los participantes que escojan una contribución o mensaje que le haya servido de mucha ayuda, la compartan y justifiquen sus razones para escogerla.

- Diseña y lleva a cabo la actividad de cierre del curso.

Es importante resaltar que no se aborda lo relacionado con los roles que el e-moderador debe desempeñar, en virtud de que se trata de otro tipo de investigación en el cual se deben considerar los patrones de actuación para cada tipología.

En síntesis, el diseño y la e-moderación en los espacios virtuales, se expresa en una gestión didáctica planificada y organizada de la enseñanza, y la comunicación didáctica, como la oportunidad de construir fuentes de información susceptibles de transformarse en conocimiento, que permite desarrollar distintas experiencias de enseñanza y de aprendizaje.

\section{Las experiencias en EVEA: un esquema referencial para su valoración a partir de la consideración del perfil de competencias}

En el estudio de las competencias especificas del docente universitario para la enseñanza en entornos virtuales, resulta significativa la reflexión en torno a las experiencias formativas de carácter virtual que se desarrollan, en el contexto de este estudio, en el nivel de pregrado de la UCV, tomando en consideración el referente teórico estudiado en páginas anteriores y los indicadores formulados.

La implantación de experiencias de formación virtual que se desarrollan en la UCV son cada vez más numerosas por lo que es necesario como afirma Carabantes Carrasco y Alves: 
teriales formativos en el componente virtual de la educación deben de tener una serie de propiedades con el fin de que sean realmente didácticos ${ }^{24}$.

La valoración de las experiencias formativas en el campus virtual con el propósito de garantizar su optimización, tal como en este espacio se concibe, se llevó a cabo con base en unos criterios de calidad que se tomaron como marco de referencia y considerando, para las dimensiones competenciales abordadas en este estudio, los componentes constitutivos y conceptuales del diseño y los rasgos que caracterizan cada una de las etapas de la e-moderación.

Tal como se ha expuesto en reiteradas oportunidades, la EaD específicamente a través de la virtualidad, se potencia a través de valiosos recursos de la Tecnología de la Información y la Comunicación. Sin embargo, como señalan Aguilar, D. y otros ${ }^{25}$ “....todos ellos por sí mismos no son garantía de aprendizaje. Son poderosos instrumentos, recursos, que deben estar enmarcados en un adecuado diseño instruccional para que resulten capaces de generarlo".

Lo antes señalado no solo destaca la importancia del diseño instruccional ajustado a las particularidades del EVEA como tarea consustancial a la acción del docente-facilitador en estos entornos, sino que pone en evidencia su estrecha relación con la calidad del producto y el proceso que se lleva a cabo. En consecuencia, el análisis de las experiencias - de acuerdo con los parámetros aquí manejados- se centra, por una parte, en el diseño y los componentes implicados en la planificación que le sirven de referencia y por otra parte, en la e-moderación (dinamización del proceso formativo).

a) Entre las consideraciones específicas que apuntan al diseño de la instrucción en los ambientes virtuales tal como aquí se concibe, resaltan las señaladas por Herrera, quien explica que estos ambientes constan de dos elementos fundamentales: los elementos constitutivos (los medios de interacción, los recursos, los factores físicos: y las relaciones psicológicas) y los elementos conceptuales referidos al concepto educativo en estos am-

24 D. Carabantes, A. Carrasco y J. Alves, "La innovación a través de entornos virtuales de enseñanza y aprendizaje”, Revista RIED, vol. 8 N 1-2 (2005), 105-126.

25 D. Aguilar et al., ¿Qué necesito aprender para ser un teleformador? Las competencias claves de la formación elearning. Programa EVA (Málaga, 2009) http://es.scribd.com/doc/25374509. 
La valoración del docente universitario en entornos virtuales: algunos descriptores claves

bientes y que se definen a través del diseño instruccional y el diseño de la interfaz.

El diseño instruccional supone una descripción de la interacción que se produce entre los protagonistas del proceso didáctico en cada uno de los momentos en que se desarrolla (independientemente del entorno al cual se refiera):

- Momento de inicio, especialmente destinado a recuperar los aprendizajes y experiencias previas relacionadas con el tema objeto de estudio, introducir el sentido e importancia del aprendizaje propuesto así como su relación con otros aprendizajes.

- Momento de desarrollo. Se caracteriza por la interacción entre el profesor y los alumnos, de estos entre sí y con los materiales de enseñanza. Este momento debe estar encaminado a desarrollar y poner en práctica las habilidades cognitivas y específicas de la disciplina además de contemplar oportunidades para la transferencia, la práctica, la construcción.

- Momento de cierre. Es un momento clave para afianzar los aprendizajes, destacar los aspectos relevantes y valorar los aspectos positivos del trabajo realizado.

El diseño de la interfaz. Se refiere a la expresión visual y formal del ambiente virtual. Es el espacio virtual en el que han de coincidir los participantes. Las características visuales y de navegación (expresadas en el menú, en el cual se puede "leer" la propuesta didáctica). Son determinantes para un adecuado desarrollo del modelo instruccional, o por el contrario, para desvirtuarla. Por ello es importante la asesoría del diseñador gráfico o infográfico en la creación de los ambientes virtuales de aprendizaje sobre la base de un buen diseño instruccional así como la asesoría del docente, quien debe velar por la expresión visual y formal del ambiente virtual para garantizar su sintonía con la planificación didáctica.

El diseño de la interfaz se traduce en el esquema general de navegación que se expresa a través del menú del entorno virtual, en el cual puede apreciarse la propuesta didáctica. Aunque el menú puede variar, en la mayoría de los casos deberán estar presentes los siguientes elementos: 
- Programa del curso

- Cronograma de actividades

- Herramientas comunicacionales

- Espacios para el intercambio de ideas y opiniones

- Espacio de socialización

- Centro de documentación y recursos adicionales

No obstante la importancia del diseño de la interfaz en un entorno virtual, en el análisis de las experiencias se privilegia lo relacionado con el diseño instruccional o diseño del proceso didáctico, en virtud de que es el elemento que justifica el sentido y el significado de la acción docente en el entorno en el cual se lleva a cabo y que orienta la definición de la interfaz. Además, en este estudio, intervienen básicamente, expertos en los componentes pedagógicos-didácticos por tratarse del aspecto focal de la investigación. En consecuencia, no se valora lo relacionado con los contenidos ni los componentes técnicos, los aspectos funcionales, estéticos y psicológicos.

Entre los aspectos generales -ya considerados en páginas anteriorespara la apreciación de la e-moderación, resultan indispensables las habilidades requeridas para proporcionar la asistencia académica y técnica adecuada, oportuna y permanente durante el proceso de formación en línea apoyándose en las herramientas comunicacionales síncronas y asíncronas, para generar un ambiente de aprendizaje cálido, sociable, motivador, interactivo, colaborativo y constructivo.

\section{CONCLUSIÓN}

Construir el perfil de competencias del docente universitario y los descriptores claves para el análisis de experiencias formativas en entornos virtuales a partir del análisis del referente teórico formulado.

La competencia en sí misma, no es conocimiento, habilidad o actitudes, sino que ella moviliza estos recursos, por ello nos identificamos con la definición de competencia como la descripción de aquello que el sujeto transfiere con sentido crítico y constructivo, que activa en el contexto de su desempeño profesional los conocimientos, capacidades, habilidades, pro- 
La valoración del docente universitario en entornos virtuales: algunos descriptores claves

cedimientos y actitudes articulados y necesarios para actuar eficazmente o de manera "competente" en situaciones específicas, únicas e irrepetibles. Su logro es dinámico y progresivo por ello se pueden ubicar diferentes niveles de logro de acuerdo con la formación y experiencia.

La mayoría de las clasificaciones estudiadas sobre competencias en EVEA, focaliza como aspecto sustancial, lo concerniente al componente pedagógico y didáctico, en virtud de que las principales funciones que tiene el profesorado en un entorno virtual es el diseño y el desarrollo de ambientes de aprendizaje que ayuden al estudiante en su proceso de formación.

Se asume que los docentes universitarios que desarrollan actividades formativas virtuales, tienen competencias básicas en las TIC (la mayor parte de la actualización docente gira alrededor de este componente y como ya se mencionó en páginas anteriores, quien se aventura a este tipo de experiencia tiene un conocimiento básico indispensable). Sin embargo, el dominio, la buena voluntad e iniciativa de aplicar las herramientas tecnológicas, no garantiza su uso adecuado desde el punto de vista didáctico en una modalidad mixta o virtual (sin menospreciar la necesaria vinculación entre el conocimiento de la disciplina, el conocimiento pedagógico y el conocimiento tecnológico). Es por ello que intentamos aproximarnos a un perfil específico, básicamente referido a las competencias didácticas (en concordancia con lo establecido por la UNESCO, que requiere el docente que se desempeña en los espacios mixtos o virtuales, sea capaz de: a) disenar acciones formativas debidamente sustentadas desde el punto de vista pedagógico, con base en todos los componentes específicos y adaptado al campus virtual en el cual se desarrolla, b) gestionar el aprendizaje mediante formas de comunicación.

Los indicadores de cada una de las dimensiones competenciales estudiadas, se formularon a partir del referente teórico analizado, sustentado en un enfoque pedagógico que caracteriza el aprendizaje centrado en el alumno (independientemente de la modalidad) como: autorregulado, significativo, conectado con la realidad, interactivo, constructivo, permanente y colaborativo. 


\section{REFERENCIAS}

Amaro de Ch. R. La docencia telemática ¿una nueva docencia? Orlando, 2009.

Aguilar, D., Fernández, A.; García, F.; García, M.; Gómez, S.; Luque, S.; Otamendi, A. y J. Haro. ¿Qué necesito aprender para ser un teleformador? Las competencias claves de la formación e-learning. Programa EVA. Málaga: 2009. http://es.scribd.com/doc/25374509/.

Bello, R. E. Educación virtual: aulas sin paredes. (Monografía) 2005. http://www.educar.org/ articulos/educacionvirtual.asp

Borges, F. La frustración del estudiante en línea. Causas y acciones preventivas. 2005. http:/ / www.uoc. edu/digithum/7/dt/esp/borges.pdf

Bustamente, H. "Redes digitales e indicadores de calidad educativa para el caso de un programa de actualización docente", en Seminario de investigación educativa: el docente y su papel en las modalidades educativas. México: Universidad Nacional Autónoma de México. http://www. distancia.unam. $\mathrm{mx} /$ foroeducativo.html

Carabantes, D.; Carrasco, A.; Alves, P. y D. Joaquim. "La innovación a través de entornos virtuales de enseñanza y aprendizaje”. Revista RIED, vol. 8 N 1 y 2. (2005): 105-126.

Cano G. y M. E. "Competences assessment in higher education La evaluación por competencias en la educación superior". Revista de Curriculum y Formación del Profesorado. Universidad de Barcelona. Barcelona: 2007 http://www.ugr.es/local/recfpro/rev123COL1.pdf

Escudero, J. M. La formación del profesorado y la garantía del derecho a una buena educación para todos. Barcelona: Octaedro, 2006.

Escudero, T. Sin tópicos ni malentendidos: fundamentos y pautas para una práctica evaluadora de calidad en la enseñanza universitaria. Zaragoza: Instituto de Ciencias de la Educación (ICE), 2009.

Gomes de O., S. "La acción docente en la educación a distancia. Competencias para la mediación en red". Revista electrónica Teoría de la Educación, Educación y Cultura en la Sociedad de la información, vol. 2 No 8 (2007): http://www.usal.es/ teoriaeducacion/rev_numero_08_02/ n8_02_oliveira.pdf

Imbernon, F. Diez ideas clave para la formación del profesorado. Nuevas ideas para la formación y el cambio. Barcelona: Graó, 2007.

Imbernon, F. et al., Análisis y propuestas de competencias docentes universitarias para el desarrollo del aprendizaje significativo del alumnado a través del e-learning y el b-learning. 2008. http://tecnologiaedu.us.es/nweb/htm/pdf/EA20070049_Dr_Francisco_Imbernon.pdf

Le Boterf, G. Ingeniería de las competencias. Barcelona: Gestión 2000/EPISE, 2000.

Luján M., M. Formación On line ¿ Tecnología vs Pedagogía? España: Learning Review, http:/ /www. learningreview.es/-ii-guia-anual-de-oferta-de-formacion-online/1561-formacion_onlineitecnologia-vs-pedagogia? format=pdf

Maspón, R. Instrumento para identificar factores que influyen en el uso de las TIC. Caracas: Universidad Central de Venezuela-Facultad de Humanidades y Educación, 2009.

Marcelo, G. Prácticas de E-learning. Las nuevas competencias en e-learning: ¿Qué formación necesitan los profesionales de e-learning? España: Octaedro, 2006. 
La valoración del docente universitario en entornos virtuales: algunos descriptores claves

Marcelo G., C. La formación de formadores: nuevos escenarios, nuevas competencias. Sevilla, España: Universidad de Sevilla, 2005.

Mishra y Koehler, Conocimiento disciplinar, pedagógico y tecnológico (Technology Pedagogical Content Knowledge TPACK). (2006-2008). http://www.wiziq.com/tutorial/71616-Mishra-ampKoehler.

Oliveira, Cervera y Martí. Learning as representation and representation as learning: a theoretical framework for teacher knowledge in the digital age. Chesapeake, VA: AACE, 2009.

Perrenoud, P. Diez nuevas competencias para enseñar. Barcelona: Graó, 2004.

Prendes, M. P.; Martínez, F.; Castañeda, L.; Torrico, M. y Mejía, R. Estudio de las competencias y demanda formativa en TIC de los docentes de las universidades bolivianas y dominicanas. Sevilla: MADEDUFORMA, 2010.

Prendes y Castañeda. Enseñanza superior, profesores y TIC. Bogotá, Colombia: Ediciones de la U, 2010.

Prendes, M. P. Competencias TIC para la Universidad Pública Española: Indicadores y propuestas para la definición de buenas prácticas, 2010. http://www.um.es/competenciastic

ProyectoTunning.Tunning Educational Structure in Europe.Informe final. Bilbao: Universidad de Deusto, 2003.

Sangrá, A. "La calidad en las experiencias virtuales de educación superior". Revista Cuadernos IRC. Barcelona, 2000. http:// www.uoc.edu/web/esp/art/uoc/0106024/sangra.html.

Sangrá, A. y González, M. La transformación de las universidades a través de las TIC: Discursos y prácticas. Barcelona-España: Educación y Sociedad Red, 2004.

Salmon, G. E-actividades. El factor clave para una formación en linea activa. Barcelona: UOC, 2002.

Silva, J. El rol moderador del tutor en la conferencia mediada por computador. Islas Baleares; EDUTEC, 2004 http://www.uib.es/depart/gte/edutec-e/revelec17/silva_16a.htm.

Silva, Gros, Garrido y Rodríguez. "Incorporación de las tecnologías de la información y la comunicación en la formación inicial docente el caso chileno". Innovación Educativa, vol. $6 \mathrm{~N}^{\circ}$ 32, (2006): 19-35.

Tobón, S. Aspectos básicos de la formación basada en competencias. Talca-México: Proyecto Mesesup, 2006.

UNESCO. Estándares de competencias en TIC para docentes, 2008). http://www.iesalc.unesco.org.ve Valdés, M. C.; Mujica, A.; Roque, A. y Darín, S. "Las competencias pedagógicas en los creativos entornos virtuales de aprendizaje universitario". Revista Electrónica de Tecnología Educativa EDUTEC N 24 (2007): http://edutec.rediris.es/Revelec2/revelec24/valdes/valdesyotros.html Zabala, A. y Arnau, L. Cómo aprender y enseñar competencias. España-Barcelona: Graó, 2007. 


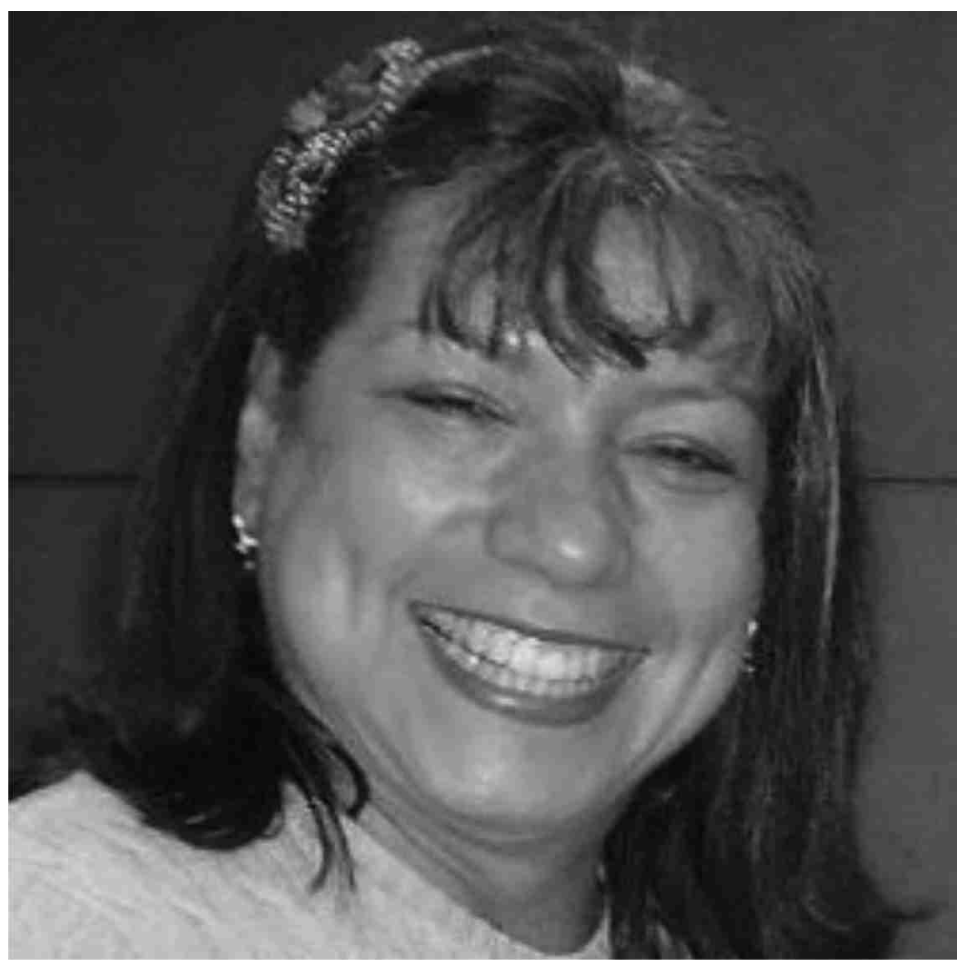

Fuente: Irama F. García.

https://www.google.com/search?hl=es\&site=imghp\&tbm=isch\&source=hp\&q=irama+g arcia\&btnG $=$ Buscar+por+imagen $\& b i w=1366 \& b i h=643 \&$ sei $=996 c U Z S E K q r D 0 A H 4 j 4$ CQDA\#imgrc=2oxhNFvQWr271 M\%3A\%3B98MKPpFsa6EGMM\%3Bhttps\%253A \%252F\%252Flh6.googleusercontent.com\%252F-_wBoijLDBp4\%252FTjEdLH8BjkE \%252FAAAAAAAAJNM\%252FP3YBiQOK2qw\%252Fs250-c-k-no\%252FProfilePhot os\%3Bhttps\%253A\%252F\%252Fplus.google.com $\% 252 \mathrm{~F} 102221975000897149354 \%$ 3B $250 \% 3 \mathrm{~B} 250$

\begin{tabular}{l|l|}
\hline $\begin{array}{l}\text { García V, Irama F.; Amaro, Rosa y Brioli, Carmen. "El perfil } \\
\text { de competencias del docente universitario en entornos virtuales: } \\
\text { algunos descriptores claves". Revista Historia de la Educación Latinoa- } \\
\text { mericana. Vol. } 14 \text { No, 19, (2012): }\end{array}$ \\
\hline
\end{tabular}

